\title{
Microwave-Assisted Chemistry - A Stock Taking
}

\author{
Bernd Ondruschka*a and Werner Bonrath ${ }^{\mathrm{b}}$
}

\begin{abstract}
In 1986 Gedye and Giguere published the first important articles on the influence of microwaves $(2.45$ $\mathrm{GHz}$ ) on syntheses undertaken in household ovens [1][2]. Over the past 20 years there have been various developments in different directions reflecting a steadily growing interest in this innovative field. This mini-overview focuses on selected details of reaction engineering in microwave chemistry.
\end{abstract}

Keywords: Energy efficiency · Microwave-assisted reactions · Process development · Qualification · Validation

\section{Introduction}

The use of high frequency waves as an alternative energy source for chemical reactions and processes has been intensively investigated in laboratories over the last 20 years but has also led to controversial discussions. A plethora of scientific publications and some recent books [3-6] dedicated to microwave-assisted chemistry covers this subject. To sum up one can conclude:

i) Up to now no reaction is known that only proceeds in the microwave field. There are always similar reactions under thermal heating conditions.

ii) Virtually every named reaction or reaction type has been studied under microwave conditions.

iii) Regardless of the impact factor of the scientific journal or its referee system, the experimental details and the microwave systems used are unfortunately usually insufficiently described. Reproducing reac-

\footnotetext{
${ }^{*}$ Correspondence: Prof. Dr. B. Ondruschka ${ }^{a}$ alnstitute of Technical Chemistry and Environmental Chemistry

Friedrich Schiller University of Jena

Lessingstr. 12

D-07743 Jena, Germany

Tel.: +493641948400

Fax: +49 3641948402

E-Mail: bernd.ondruschka@uni-jena.de

bDMS Nutritional Products

Research \& Development

P.O. Box 3255

$\mathrm{CH}-4002$ Basel

E-Mail: werner.bonrath@dsm.com
}

tion parameters such as conversion, selectivity and yield is often only possible with difficulty or even impossible because of incomplete description of the experiments.

As a counterpart to thermal heating, the use of microwave irradiation for the specific activation of chemical reactions requires an extended theoretical knowledge. There are empirical measurement problems and physical problems when using microwaves for synthetic chemistry. This makes the transfer of classical chemical synthesis to technical engineering sciences unavoidable. The fact that more measurement technology will be required is contradictory to current trends in miniaturisation and simplification observed in organic synthesis. While the analytical equipment (NMR, GC) used is often reported extensively and in detail, the exact description of the microwave devices employed are rarely documented. The type of the microwave device, often a domestic microwave oven, is not even mentioned. The power used is described in terms of 'full power' or only the preset power step is given. Thus, it is impossible to draw safe conclusions about the achieved temperatures. In addition, reactions performed are often only compared to literature data from reactions that were performed under completely different or difficult to compare conditions. For a scaleup of laboratory results (mmol scale), it is necessary to know the experimental details (e.g. apparatus, reaction protocol). This is a general rule for all process developments, and not only connected to chemistry under microwave conditions.

Since about 1986, the technical developments, e.g. possibilities for temperature and pressure measurement, continuous processes, unpulsed energy entry, micro- wave leak sensors, in specialized systems that are adapted to chemical synthesis have extended the possibilities of microwave-assisted reaction engineering. With these new benefits, there is a growing interest in scaling-up this method.

\section{Principles of Green Chemistry}

The condensed principles of green chemistry are summarized in [7] (Scheme). One goal is to decrease energy input. The development of new synthetic methodology under microwave assistance is an important area of research with growing attractiveness.

\begin{tabular}{|ll|}
\hline $\mathbf{P}$ & Prevent wastes \\
$\mathbf{R}$ & Renewable materials \\
$\mathbf{O}$ & Omit derivatization steps \\
D & Degradable chemical products \\
$\mathbf{U}$ & Use safe synthetic methods \\
$\mathbf{C}$ & Catalytic reagents \\
$\mathbf{T}$ & Temperature, pressure ambient \\
$\mathbf{I}$ & In-process monitoring \\
$\mathbf{V}$ & Very few auxiliary substances \\
$\mathbf{E}$ & Factor, maximise feed in product \\
$\mathbf{L}$ & Low toxicity of chemical products \\
$\mathbf{Y}$ & Yes, it is safe! \\
\hline
\end{tabular}

Scheme. Condensed principles of Green Chemistry (cf. [7])

Green chemistry needs to be combined with more environmentally friendly technologies if step-change improvements are necessary in chemical manufacturing processes. Synthetic chemists have traditionally not been adventurous in their choice of the reactor types: The familiar round-bot- 
tomed flask with (mechanical, magnetic) stirrer remains almost always the automatic choice for most, even when the planned chemistry is innovative, e.g. the use of a non-volatile solvent (ionic liquids) or an immobilized homogeneous or heterogeneous catalyst as an alternative to a soluble reagent. However, an increasing number of research articles describing green chemical (engineering) reactions are based on alternative reactors.

Energy questions have been often (somewhat) neglected in the calculations of resource utilization for a chemical process. Batch processes based on scale-up reaction pots can run for many hours or even days to maximize yield and often suffer from poor mixing and heat transfer characteristics. As the cost of energy increases and greater efforts are made to control emissions associated with generating energy, energy consumption will increasingly become one of the most important part of green chemistry metrics calculations. This will open the future door not only to better designed reactors such as those described below but also to use of alternative energy sources.

Microwave-assisted chemistry is based on the use of intensive directed irradiation and its purposeful application can lead to following advantages:

- to short heating times,

- to a wide temperature and pressure range,

- to accumulation of inserted energy in the system,

- to inversion of heating flux in the reactor,

- to high(er) energy efficiency,

- to sophisticated measurement and safety technology, and

- to modular systems which allow changing from $\mathrm{mg}$ up to $\mathrm{kg}$ scale.

\section{Energy Efficiency of Microwave- assisted Reactions}

In general, discussion about 'energy efficiency' should always relate to comparable parameters. The question whether microwave energy can be used for the activation of chemical reactions more efficiently cannot be answered spontaneously. In most of the published work investigations were only carried out with very small amounts of reaction mixture, which were then irradiated with comparatively high power (300 to $1000 \mathrm{~W}$ ). A factor that describes the efficiency of the microwave input was too rarely included in investigations or discussions [8]. Such a discussion was and will be neglected as long as reactions are carried out on a miniature scale ( $\mathrm{mmol})$. Considering a scale-up ( $\mathrm{mmol} \rightarrow \mathrm{mol} \rightarrow \mathrm{kmol})$, the limitation of this approach becomes obvious [9][10].
As often within the reactor design the reaction field homogeneity within the microwave applicator $(c f$. the phenomenon of field distributions under sonochemical or photochemical conditions!) is an essential criterion. The results observed in domestic microwave reactors demonstrate clearly inhomogeneity of the irradiation fields. Most important problems in scale-up from microwave cavities are for that reason the:

- energy efficiency,

- uniformity of heating in the irradiated zone, and

- safe monitoring and control of reaction.

The control of the energy input plays an essential role in reaching the predefined reaction conditions for the overall treatment of reaction mixtures in organic chemistry. In household microwave devices, reaction time and the power irradiated during this time can be varied preferably as reaction parameters. Thus, the measurement of temperature is undetermined. A possible but insufficient method to control the temperature is the on- and off-switching of the microwave field within a given time interval. In modern commercial laboratory microwave systems, however, computer controls, which allow setting the upper temperature or pressure as limiting parameters, are state of the art. This feature is important with regard to safety aspects of handling chemicals and crucial for the reproducibility and the scale-up of reactions under good management conditions. After reaching the preset parameters, the energy input is reduced to a level necessary to maintain the preset values. This power control contributes essentially to enhancement of efficiency of the power input by microwaves. The microwave energy proceeds almost free of loss through a microwave-transparent reactor walls into the reaction mixture and is then converted into heat, which is accumulated in the reaction mixture and stays there because reactor materials are also good heat insulators

This circumstance precisely illustrates the advantage of power input by microwaves. High power can be applied to reaction mixtures which are able to absorb microwaves in a controlled and fast manner. Other reaction parameters, such as the usage of real equipment and reaction types [10], play just as an important role. This fact requires that synthetic chemists have to be well educated in the field of chemical engineering.

Working with unpulsed microwave radiation exhibits advantages among other things for refluxing, distilling and photoreactions under microwave irradiation. Sensitive substances should not be overheated. The disadvantage of unpulsed power supplies in comparison to conventional systems is their higher technical complexity.
The use of unpulsed power supplies will result in advantages on the long run, also with respect to energy efficiency.

At this point, it is necessary to discuss the term 'penetration depth' in more detail. The microwave cavity of the presently biggest synthesis microwave system comprises approximately $100 \mathrm{l}$ and allows for reactions on the pilot plant-scale. Microwaves penetrate from all sides into the sample and lead to a mostly homogeneous energy input, which must be additionally improved by stirring the samples. Considering the penetration depth of microwaves in water of $1.4 \mathrm{~cm}$ at $25{ }^{\circ} \mathrm{C}$ and $5.7 \mathrm{~cm}$ at $95{ }^{\circ} \mathrm{C}$, the reactor dimensions are limited [11]. More precise prediction for organic solvents is hardly possible at the moment due to a lack of data. However, since water has a comparatively high dielectric coefficient, higher penetration depths can be expected at ambient temperature for the majority of substances used in organic synthesis.

\section{Process Development of Microwave-assisted Reactions}

A common requirement associated with the introduction of new technologies is the possibility to scale-up the respective process, first to a miniplant scale and then to production scale.

Microwave systems that are commercially available at present were initially developed for chemical digestion. This limited the size of reactors that could be used for chemical synthesis. With effective volumes of 25-50 ml, in rare cases $250 \mathrm{ml}$, reactions on the $10-50 \mathrm{mmol}$ scale could be performed. The concept of solvent-free reactions is discussed in detail in [12]. The further development of microwave systems for applications in organic chemistry leads in two directions.

One direction is the development of small devices or devices that are tailored to a special application. The small devices allow for the reaction on mmol scale in a short time (several minutes) with comparatively high power input; they possess a small microwave cavity $(\leq 11)$, and have a reactor tool installed directly in the waveguide, in which often only small and closed vessels can be used. These systems are advantageous for organic chemists for yes-or-no-answers with respect to the experimental results. If the research scope is extended to questions about the reproducibility, the reaction kinetics, or the increase of the synthesis to afford 0.1 mol product (factor 100), these devices will fail. This product line is developed by Biotage (Sweden) with their differently automated products of the EMRYS line, by the defunct Prolabo (France) with monomode systems of the 'Synthewave' series, and CEM (USA) with their 'Discovery' se- 
ries [10]. CEM also produces a wide range of multimode devices, which are mainly used for sample preparation (decomposition, ashing) and sometimes also for carrying out organic syntheses [4].

The second direction is the design of different reactor tools that can be integrated in the same basic system (ETHOS system, MLS/Milestone) [12]. The ETHOS system allows the development of a concept for the comparative transition of classically heated reactions into the microwave field. The usage of reactors for different requirements and applications allows for a flexible reaction engineering in which all reaction parameters can be precisely documented. Simultaneously, it is possible, through the exact reproduction of conventional conditions, to compare classical and microwaveassisted reactions with each other and thus to realise the advantages of the microwave processing without speculations. With this construction kit, reactions from the mmol scale to the mol scale can be carried out. Furthermore, the transition from batch operation to continuous operation is also imaginable, and has already been described for some reaction types [8][9][12]. Further, a pilot plant device was derived from the base mode in which first studies on the real scale-up were performed [8]. The ETHOS 4000/4001 devices can already process reaction mixtures of 5-10 kg per hour and are thus suitable for the production of highpriced fine chemicals, e.g. pharmaceuticals.

Manufacturers and chemical engineers have started a concept of the transition of conventional reactions in the microwave field, which tries to find solutions through 'scale-down' and 'numbering-up' approaches as well as through 'scale-up' concepts.

The goal of all these investigations is to obtain a holistic view and to question all reaction parameters employed so far in order to discover new innovative ways for carrying out known reactions. Therefore, the description of all reaction parameters is absolutely necessary.

\section{Sustainable Aim of Microwave- assisted Reactions}

Microwave processing should used to accelerate reactions that suffer from disadvantageous reaction parameters (i.e. long reaction times at comparatively low temperatures, solvent use, excess components, etc.). A further goal for process improvement is to transfer a batch (discontinuous) to a continuous operation after they have undergone a process analysis. For this purpose, usually the first step should be to repeat the known reaction conditions used in the conventional reactions under microwave influence. Often a comparable ex- perimental setup (reflux apparatus) is used. Starting from this point, all conventional reaction conditions must be re-evaluated. The introduction of a new(er) technology in organic technical synthesis allows for the questioning of old preparation protocols and notes. It would be advantageous to produce a check list for each reaction that critically questions the known synthetic protocols, analyses, and provides potential new solutions. When studying process development a variety of parameters should be addressed. A list of questions follows:

- What is the required temperature for this specific reaction?

- Are all (which) compounds of the reaction mixture thermally stable?

- Is the reaction exo- or endothermic? (Is the energy well balanced?)

- Do secondary reactions occur? (Why?)

- Is a solvent imperatively necessary? (Why?)

- How is the work-up? (Which work-up processes require how much (heat) energy?)

- Is a catalyst required? (Which catalyst and in what quantity?)

- Can the catalyst be recycled? Is the recycling advantageous?

- Which reaction conditions influence each other?

- Is the stoichiometry optimal?

- Are waste materials and/or by-products formed and how can they be handled?

- Do toxicological/ecotoxicological data exist?

This list can be effortless extended on the basis of the rules for sustainable development [13] in all areas. Microwave technology allows for the investigation of these questions with the present technology by changing reaction parameters and checking old reaction protocols.

The question of generalisation, reproducibility, and scale-up of the selected reactions will always imply the question about the reaction conditions. The reaction medium plays a much more important role for this kind of power input compared to classical heating. Besides the molecule mass, the polarity of the reaction mixture is essential for the absorption of microwave power. Since dielectric constants (static and dynamic) of compounds are often unknown, more problems arise and require the contact with neighbouring disciplines, for example with electrical engineering.

From the arguments and facts presented in this short overview, it becomes obvious that the concept of a 'comprehensive chemistry' is not useful in the case of microwaveassisted reactions. The primary literature reflects the results of microwave-assisted reactions and processes in the same incomplete fashion as it does for conventional syntheses. However, the dependency of the reaction engineering on technical param- eters is even higher for microwave-assisted reactions. Therefore, an improvement of the description of reaction parameters is crucial. These remarks lead to the development of a draft for a general experimental protocol for microwave-assisted reactions and processes. Its introduction and application in the sense of sustainability are given in [8][9].

\section{Qualification and Validation of Reactors and Results}

Qualification of technical devices and validation of reactions and processes are fundamental factors for the introduction of new techniques and technologies and methods in the laboratory as well as in industry. In the pharmaceutical industry, product development and production is unimaginable without qualification and validation. The definition and reproducibility of system parameters and reaction conditions are pivotal elements in addition to the ability to keep exact records of reactions and processes. Unfortunately, quality management is often neglected for basic chemistry research and process development in academia. Often it is also completely omitted from the chemistry education. The consideration of both a. m. criteria are the best guarantor for emotionless discussions on the existence of microwave effects [9].

Recent literature on microwave-assisted chemistry has described a multitude of different effects on chemical reactions and processes attributed to microwave radiation. As mentioned published experimental protocols are often insufficient and do not allow reproduction of the obtained results. Important factors required for qualification and validation such as exact records, reproducibility, transparency of reactions/processes are commonly not reported, which poses a serious drawback for the industrial development of microwave-assisted reactions and processes for the synthesis of fine chemicals, intermediates and pharmaceuticals. Technical microwave devices for synthetic chemistry have been on the market for a while and should allow comparative investigations to be carried out at set parameters. These investigations allow a better assessment of the observed effects. Furthermore, it is possible to obtain a better insight into the often discussed (non-thermal) microwave effects from these experiments. Technical microwave systems represent an important first step toward the use of microwave energy for technical syntheses. The actual scale-up of synthetic reactions in the microwave is, however, still to be undertaken. Comparisons between microwave systems with different technical parameters should provide a measure for the qualification of the employed systems, which in turn 
is important for the validation of reactions and processes carried out in such commercial systems.

\section{Conclusions}

'Simple heating with microwaves' is nowadays common laboratory practice for many preparative chemists. Microwave systems with integrated on-line control guarantee safe operation and open a vast field of applications, even feeble on the technical scale. In the context of this overview, it seems worthwhile to critically assess all reaction parameters for syntheses and separations and to coordinate them with each other. In other words besides the obvious need to learn more about microwave-assisted reactions, there are also promising challenges ahead.

For technical applications and implementation of a new method, it is necessary to have equipment available. For application in a synthetic pathway not only criteria like E-factor or atom economy are important, the equipment factor also plays an important role:

\section{Implementation $=$ Atom Economy $\times$ (weighted) E-factor $\times$ Equipment factor}

For example, if the equipment is available around the world without any restrictions, the equipment factor is 1 . In the case of microwaves this factor is around 0.3 because for large-scale industrial production (several 1000 tons) no equipment exists. The hope exists that the use of microwave irradiation will be typically applied in enterprises and chemical industry as already nowadays in laboratories. There are some glimmers of hope that the 'Bunsenbrenner' of the 21 st century will become a valued and widely used tool for chemists and engineers.

Received: May 5, 2006
[1] R. Gedye, F. Smith, K. Westaway, H. Ali, L. Baldisera, L. Laberge, J. Rousell, Tetrahedron Lett. 1986, 27, 279.

[2] R.J. Giguere, T.L. Bray, S.M. Duncan, G. Majetich, Tetrahedron Lett. 1986, 27, 4945.

[3] a) 'Microwaves in Organic Synthesis', Ed. A. Loupy, 1st ed., Wiley-VCH, Weinheim, 2002; b) 'Microwaves in Organic Synthesis', Ed. A. Loupy, 2nd ed., Wiley-VCH, Weinheim, 2006.

[4] B.L. Hayes, 'Microwave Synthesis - Chemistry at the Speed of Light', CEM Publ., Matthews (NC), 2002.

[5] C.O. Kappe, A. Stadler, 'Microwaves in Organic and Medicinal Chemistry', Wiley-VCH, Weinheim, 2005.

[6] 'Microwave Assisted Organic Chemistry', Ed. J.P. Tierney, P. Lidström, Blackwell Publ., Oxford, 2005.

[7] S.L.Y. Tang, R.L. Smith, M. Poliakoff, Green Chem. 2005, 7, 761.

[8] M. Nüchter, B. Ondruschka, W. Bonrath, A. Gum, Green Chem. 2004, 6, 128.

[9] M. Nüchter, B. Ondruschka, D. Weiss, R. Beckert, W. Bonrath, A. Gum, Chem. Eng. Tech. 2005, 28, 871.

[10] B. Ondruschka, W. Bonrath, D. Stuerga, 'Development and design of reactors in laboratory and pilot scale in microwaveassisted chemistry', in: [13].

[11] D.R. Baghurst, D.M.P. Mingos, J. Chem. Soc. Chem. Commun. 1992, 674.

[12] M. Nüchter, U. Müller, B. Ondruschka, A. Tied, W. Lautenschläger, Chem. Eng. Tech. 2003, 26, 910.

[13] 'Green Separation Processes, Fundamentals and Applications', Ed. C.A.M. Afonso, J.G. Crespo, Wiley-VCH, Weinheim, 2005. 